\title{
lyengar Yoga Increases Cardiac Parasympathetic Nervous Modulation Among Healthy Yoga Practitioners
}

\author{
Kerstin Khattab ${ }^{1}$, Ahmed A. Khattab ${ }^{1}$, Jasmin Ortak ${ }^{2}$, Gert Richardt ${ }^{1}$ and \\ Hendrik Bonnemeier ${ }^{2}$ \\ ${ }^{1}$ Herz-Kreislauf-Zentrum Segeberger Kliniken GmbH, Bad Segeberg and ${ }^{2}$ Medizinische Klinik II,
Universitätsklinikum Schleswig-Holstein Campus Lübeck, Lübeck, Germany
}

\begin{abstract}
Relaxation techniques are established in managing of cardiac patients during rehabilitation aiming to reduce future adverse cardiac events. It has been hypothesized that relaxationtraining programs may significantly improve cardiac autonomic nervous tone. However, this has not been proven for all available relaxation techniques. We tested this assumption by investigating cardiac vagal modulation during yoga.We examined 11 healthy yoga practitioners ( 7 women and 4 men, mean age: $43 \pm 11$; range: $26-58$ years). Each individual was subjected to training units of $90 \mathrm{~min}$ once a week over five successive weeks. During two sessions, they practiced a yoga program developed for cardiac patients by B.K.S. Iyengar. On three sessions, they practiced a placebo program of relaxation. On each training day they underwent ambulatory $24 \mathrm{~h}$ Holter monitoring. The group of yoga practitioners was compared to a matched group of healthy individuals not practicing any relaxation techniques. Parameters of heart rate variability (HRV) were determined hourly by a blinded observer. Mean RR interval (interval between two R-waves of the ECG) was significantly higher during the time of yoga intervention compared to placebo and to control $(P<0.001$ for both). The increase in HRV parameters was significantly higher during yoga exercise than during placebo and control especially for the parameters associated with vagal tone, i.e. mean standard deviation of NN (Normal Beat to Normal Beat of the ECG) intervals for all 5-min intervals (SDNNi, $P<0.001$ for both) and root mean square successive difference (rMSSD, $P<0.01$ for both). In conclusion, relaxation by yoga training is associated with a significant increase of cardiac vagal modulation. Since this method is easy to apply with no side effects, it could be a suitable intervention in cardiac rehabilitation programs.
\end{abstract}

Keywords: yoga-Iyengar Yoga-heart-rate-variability-cardiac rehabilitation

\section{Introduction}

Heart rate variability (HRV) has been established as a noninvasive tool to study cardiac autonomic activity. Reduced HRV has been established as a predictor for increased risk of cardiac mortality and sudden cardiac death (1-6) especially in patients after myocardial infarction. Several relaxation techniques have been established in the

For reprints and all correspondence: Kerstin Khattab, MD, Segeberger Kliniken GmbH, Am Kurpark 1, 23795 Bad Segeberg, Germany.

E-mail: kerstinkhattab@t-online.de management of patients during cardiac rehabilitation aiming to reduce future cardiac events via cardiac autonomic nervous activity. It has been hypothesized that relaxation training programs may improve the cardiac autonomic nervous tone. However, this has not been proven for all available relaxation techniques, such as yoga. Since more than 60 years, B.K.S. Iyengar has been working therapeutically with patients after myocardial infarction. His method offers more than other techniques for relaxation: the sequence of yoga asanas (postures) and the individuality they are performed with, are adjusted to 
the severity of myocardial infarction, the stage of recovery and the clinical condition of the patient. Props are used, so that the strain of exercise can be regulated or stopped if desired. We investigated whether this method has a positive influence on the cardiac vagal modulation compared to a similar setting of conventional relaxation and exercise techniques among healthy yoga practitioners.

\section{Methods}

\section{Subjects}

We examined 11 healthy yoga practitioners (7 women and 4 men, mean age: $43 \pm 11$; age range: $26-58$ years). They were experienced practitioners with at least 3 years of regular practice of Iyengar Yoga; four of them were certified teachers of Iyengar Yoga. Each individual was subjected to five training units each of $90 \mathrm{~min}$ at the same time of the day (around 12.00-13.30 p.m.) once a week over five successive weeks. During two sessions, they practiced Iyengar Yoga; on three sessions they practiced a placebo program of relaxation that consisted of resting on the floor and park walking. To avoid misinterpretations of our findings due to interindividual and circadian variability (7), the yoga practitioners themselves served as an intraindividual control group. The group of yoga practitioners was also compared to an age and gender matched group of healthy individuals without evidence of cardiovascular disease who have not been practicing any relaxation techniques $(n=11)$, to identify long-term effects. All volunteers gave informed consent for scientific use of their Holter information.

\section{Intervention}

Before the actual examination started, the yoga practitioners had 3-5 sessions where they practiced the sequence of yoga asanas chosen for the intervention to make them familiar with the program. Since the yoga practitioners were experienced, the program, which was taken from the work of B.K.S. Iyengar, addressed patients after myocardial infarction already at an advanced stage of recovery. The program started with about $15 \mathrm{~min}$ resting poses, continued by $60 \mathrm{~min}$ standing poses, backbends and inverted poses and ended with another $15 \mathrm{~min}$ of resting poses. The sequence of asanas is shown in Table 1. Usually a training program for cardiac patients ends with the asana Bhismacharyasana; here however we changed the series to end the program with Shavasana (lying on the floor) to simulate the final resting phase of the placebo program. Asanas were conducted by a certified teacher for Iyengar Yoga (K.K.). All asanas of B.K.S. Iyengar's work with cardiac patients after myocardial infarction focus on opening the chest; therefore in standing poses a trestle
Table 1. Sequence of asanas (yoga-postures) performed by 11 yoga students during time of intervention

Shavasana with support (corpse pose, see Fig. 1a)

Supta Baddha Konasana with support (supine, bound angle pose)

Purvottanasana on bench and support (intense stretch of the front of

the body, see Fig. 1b)

Trikonasana with a trestle (triangular standing pose)

Parshvakonasana with a trestle (lateral angle standing pose)

Ardha Chandrasana with a trestle (half moon standing pose, see Fig. 1c)

Prasarita Pardottasasana, concave back (spread legs, intensely streched)

Bharadvajasana, sitting on chair, hands on trestle (twisting pose named after Bharadvaja)

Adho Mukha Shvanasana with ropes (downward facing dog pose)

Shirshasana (headstand)

Viparita Dandasana with bench (inverted stick pose)

Dhanurasana with or without support (upward bow pose, see Fig. 1d)

Sarvangasana with chair (shoulderstand)

Halasana with support (plough pose)

Bhismacharyasana with support (backbend named after Bhisma, see Fig. 1e)

Setubandha Sarvangasana with support (bridge pose)

Viparita Karani on Setubandha Bench (inverted lake pose)

Shavasana with support (corpse pose, see Fig. 1a)

was used and in supine position a special support for the thoracic spine was applied (e.g. wooden plank and small heart brick), see Fig. 1 a-e. The exact description of the exercises is beyond the scope of this article. Details have been described by B.K.S. Iyengar elsewhere (8-9). The placebo program was designed to be comparable to the yoga intervention and consisted of about $15 \mathrm{~min}$ resting on the floor in supine position, 60 min park walking and again 15 min of resting on the floor. The group was familiar with this kind of activity. During park walking or resting, the trainees were also under guidance of the yoga instructor, and were taught to relax certain muscle groups.

\section{Data Collection}

During five successive weeks, once a week all volunteers underwent 24-h ambulatory ECG monitoring with two-channel time-tracking Holter recorders (Tracker II, Reynolds, Herford, UK). The Holter Recording was initiated between $11 \mathrm{a} . \mathrm{m}$. and $12 \mathrm{p} . \mathrm{m}$. At around 12 p.m., the intervention program started. During three sessions, they underwent the placebo program; during two sessions the yoga program was conducted. All Holter recordings were manually edited by an experienced physician (H.B.) for exclusion of artifacts and premature beats. The physician was unaware of the group and the intervention. One RR interval (interval between two $R$ waves of the ECG) before and five-RR intervals after an atrial or ventricular premature beat were eliminated from the analysis. A minimum of $22 \mathrm{~h}$ of analyzable data and a minimum of $90 \%$ of analyzable NN (Normal Beat to Normal Beat of the ECG) intervals were required for a tape to be accepted as valid. The median duration of the recordings was $24 \mathrm{~h}$, with $96 \%$ of valid analyzable NN intervals. There was no drop out. 
(a)

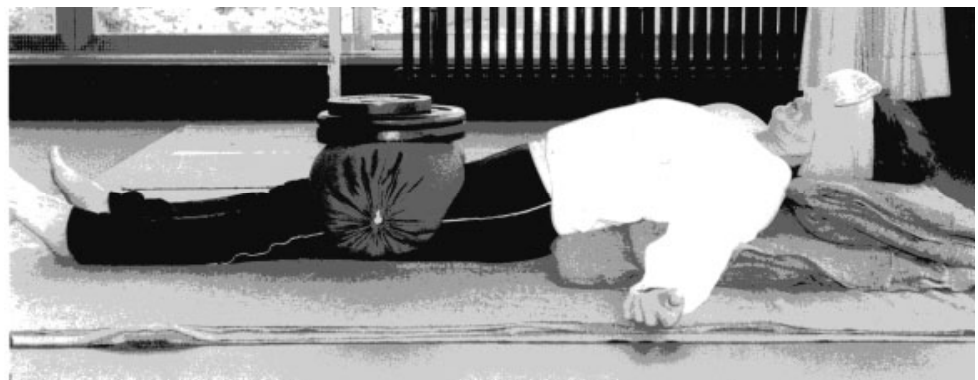

(b)

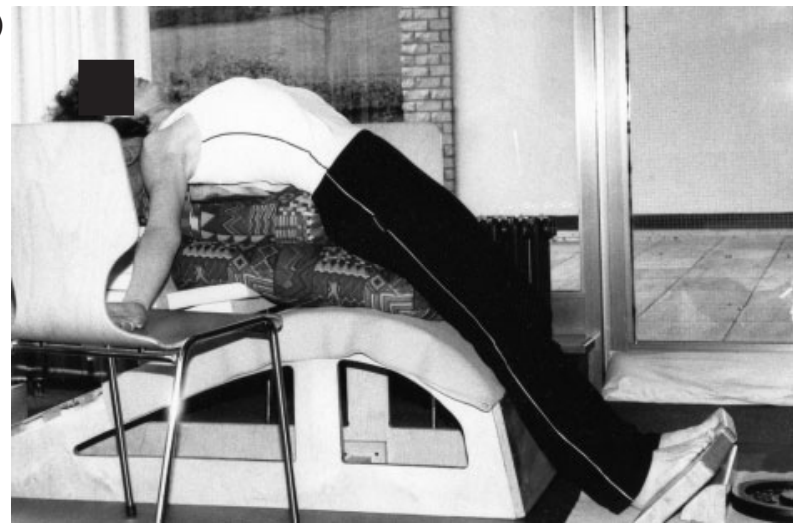

(c)

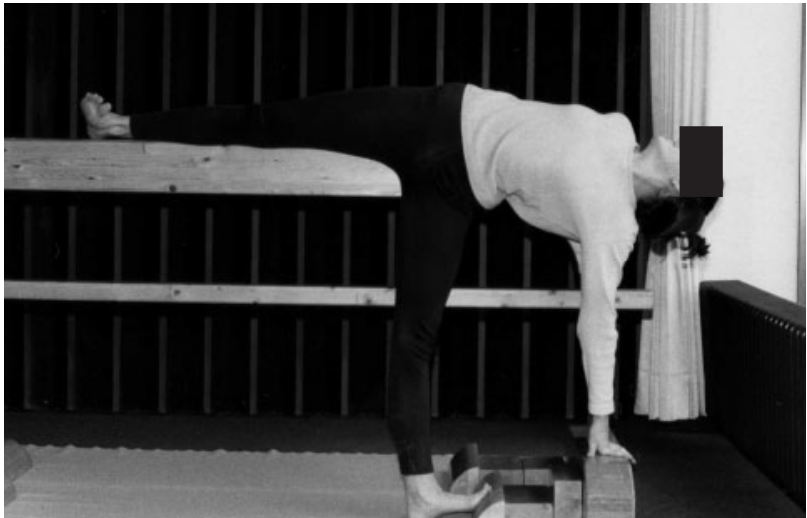

(d)

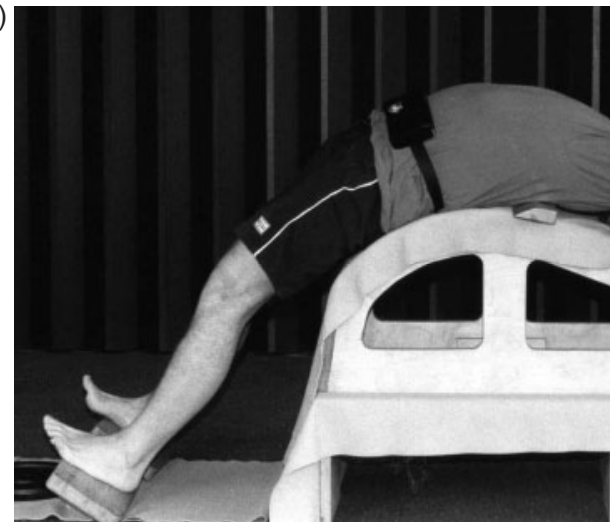

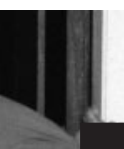
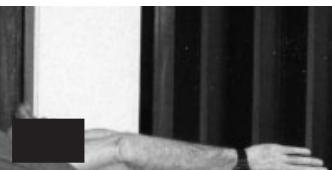

(e)

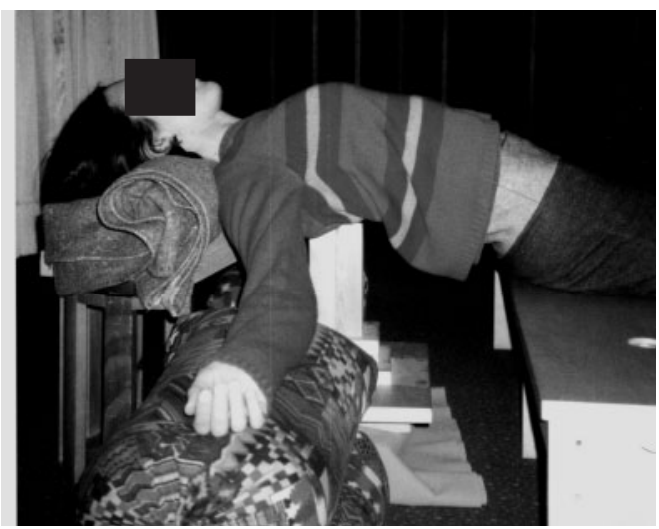

Figure 1. (a) Shavasana/corpse pose. (b) Purvottanasana on bench and support/intense stretch of the front of the body. (c) Ardha Chandrasana with a trestle/half moon standing pose. (d) Urdhva Dhanurasana with support/upward bow pose. (e) Bhismacharyasana with support/backbend named after Bhisma.

\section{Analysis of HRV}

For each subject, time-domain HRV was measured according to the Task Force of ESC and NASPE (2) using a Pathfinder digital analysis system (Delmar Reynolds). Mean RR interval and the following HRV parameters were calculated as hourly values and as $24-\mathrm{h}$ values: square root of the mean of the sum of the squares of differences between adjacent NN intervals (rMSSD), standard deviation of NN intervals (SDNN), mean standard deviation of NN intervals for all 5-min segments (SDNNi), standard deviation of the averages of $\mathrm{NN}$ intervals for all 5-min segments (SDANN), absolute count of adjacent successive $\mathrm{NN}$ intervals differing by $>50 \mathrm{~ms} / \mathrm{h} \quad(\mathrm{sNN} 50)$ and geometrical triangular index (TI).

\section{Statistical Analysis}

Statistical analyses were conducted with a commercially available software package (SPSS version 12.0; SPSS Inc). Comparisons between groups were performed utilizing a Mann-Whitney U-test. Multiple comparisons were done by Bonferroni corrected analysis of variance for repeated measures. Consecutively, an alpha corrected paired Student's $t$-test was performed for interval-tointerval comparisons. HRV-parameters were tested for normal distribution with the Komolgorov-Smirnov 
Table 2. Baseline criteria of healthy yoga practitioners subjected to yoga or placebo and their matched healthy controls

\begin{tabular}{lll}
\hline & $\begin{array}{l}\text { Yoga } \\
\text { practitioners } \\
(n=11)\end{array}$ & $\begin{array}{l}\text { (Controls } \\
n=11)\end{array}$ \\
\hline Male (\%) & 36.4 & 36.4 \\
$\begin{array}{l}\text { Age range (years) } \\
\begin{array}{l}\text { Smoker, hypertension, diabetes, } \\
\text { history of cardiovascular disease (\%) }\end{array}\end{array}$ & $26-58$ & $26-57$ \\
\hline
\end{tabular}

goodness-of-fit test for normality. All parameters but sNN50 were normally distributed. Although natural logarithmic transformation could diminish the skewness of the distributions of sNN50, data was not transformed with regard to the lack of comparability with previous published data. HRV-data are presented as mean values \pm standard deviation. Statistical significance was set up at $P<0.05$.

\section{Results}

\section{HRV Parameters Outside Intervention Time}

The baseline criteria of yoga practitioners and the matched control group are shown in Table 2. There were no significant differences regarding hourly mean values of RR interval and parameters of $H R V$ outside the intervention time.

\section{RR Interval and Parameters Associated to the Vagal Tone During Intervention}

Mean RR interval was significantly higher during the time of yoga intervention compared to placebo and to control $(865 \pm 119 \mathrm{~ms} ; 746 \pm 86 \mathrm{~ms} ; 753 \pm 115 \mathrm{~ms}$, respectively, $P<0.001$ for both). Increase in the parameters of HRV was significantly higher during yoga exercise than during the placebo program and control especially for parameters associated to vagal tone [mean standard deviation of $\mathrm{NN}$ intervals for all 5-min intervals (SDNNi) $86.9 \pm 16$ versus $62.9 \pm 53.3 \pm 18(P<0.001$ for both); root mean square successive difference rMSSD $37.3 \pm 10$ versus $30.1 \pm 9$ versus $24.1 \pm 12(P<0.01$ for both)].

\section{Estimates of Overall HRV and Parameters Associated with Physical Activity During Intervention}

Estimates of overall HRV were significantly higher regarding the geometrical TI during yoga compared to placebo and control at time of intervention $(26.5 \pm 6$ versus $24.6 \pm 8$ versus $17.6 \pm 6 ; P<0.001$ for both). Standard deviation of NN interval (SDNN) was not significantly different during yoga exercise compared to the placebo program, but was significant for both compared to the control at time of intervention $(129.6 \pm 22$ versus $130.7 \pm 32$ versus $78.7 \pm 26 P<0.001)$. Standard Deviation of the averages of NN intervals for all $5 \mathrm{~min}$ segments (SDANN) - a long-term parameter associated with physical activity - was higher during the placebo program of resting and park walking compared to the yoga program $(90.8 \pm 32$ versus $116.6 \pm 33.5$; $P<0.01)$ and significantly higher for both yoga and placebo compared to the control $(P<0.001)$, results are demonstrated in Fig. 2 a-g and Table 3.

\section{Discussion}

This study demonstrates that relaxation by yoga training is associated with a significant increase of cardiac vagal modulation among healthy yoga practitioners. Since this method is easy to apply with no side effects, and leads to a deep physical and mental relaxation, it could be a suitable intervention during cardiac rehabilitation to shift the autonomic balance towards an increase of vagal activity and possibly decrease cardiac mortality. La Rovere et al. (10) showed that exercise training by bicycle ergometry, an established training method in cardiac rehabilitation programs, increases vagal activity in patients after myocardial infarction, investigating baroreflex sensitivity as an autonomic marker. However, they reported that exercise training alone does not seem to be the only determinant of improved

\footnotetext{
Figure 2. (a) Mean hourly results of RR-interval ECG-recordings among 11 Yoga students and a matched control group. The red arrow marks time of intervention of the yoga group (Yoga or Placebo). *** Yoga versus Placebo $P<0.001$, **Yoga versus Control $P<0.01$. (b) Mean hourly results of SDNN ECG-recordings among 11 Yoga students and a matched control group. The red arrow marks time of intervention of the Yoga group (Yoga or Placebo). ${ }^{* * *}$ Yoga versus Control $P<0.001$, ***Placebo versus Control $P<0.001$. (c) Mean hourly results of SDNNi ECG-recordings among 11 Yoga students and a matched control group. The red arrow marks time of intervention of the Yoga group (Yoga or Placebo). ${ }^{* * *}$ Yoga versus Placebo $P<0.001,{ }^{* * *}$ Yoga versus Control $P<0.001$. *Placebo versus Control $P<0.05$. (d) Mean hourly results of SNN50 ECG-recordings among 11 Yoga students and a matched control group. The red arrow marks time of intervention of the Yoga group (Yoga or Placebo). **Yoga versus Placebo $P<0.01$, **Yoga versus Control $P<0.01$. (e) Mean hourly results of SDANN ECG-recordings among 11 Yoga students and a matched control group. The red arrow marks time of intervention of the Yoga group (Yoga or Placebo). **Yoga versus Placebo $P<0.01$, ***Yoga versus Control $P<0.001$, ***Placebo versus Control $P<0.001$. (f) Mean hourly results of rMSSD ECG-recordings among 11 Yoga students and a matched control group. The red arrow marks time of intervention of the Yoga group (Yoga or Placebo). **Yoga versus. Placebo $P<0.01$, ** Yoga versus Control $P<0.01$, *Placebo versus Control $P<0.05$. (g) Mean hourly results of TI ECG-recordings among 11 Yoga students and a matched control group. The red arrow marks time of intervention of the Yoga group (Yoga or Placebo). ${ }^{* * *}$ Yoga versus Placebo $P<0.001$, *** Yoga versus Control $P<0.001$, *Placebo versus Control $\mathrm{P}<0.0$.
} 
(a) RR Interval

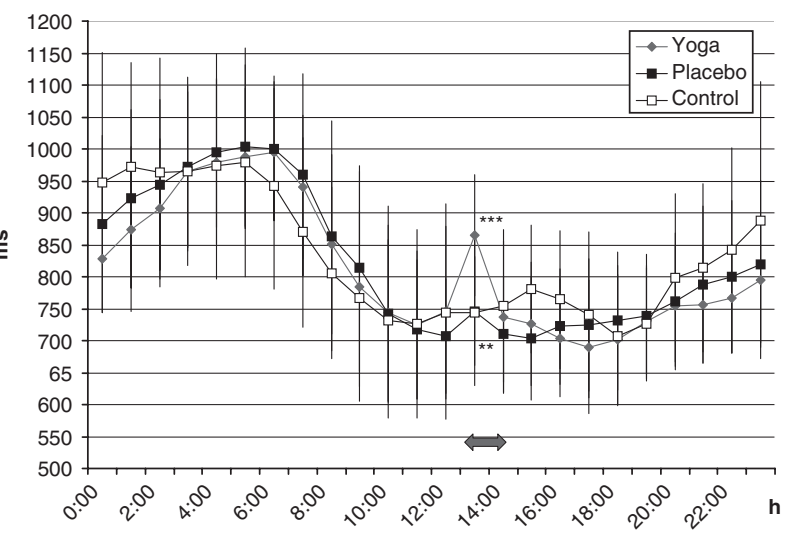

(c) SDNNi

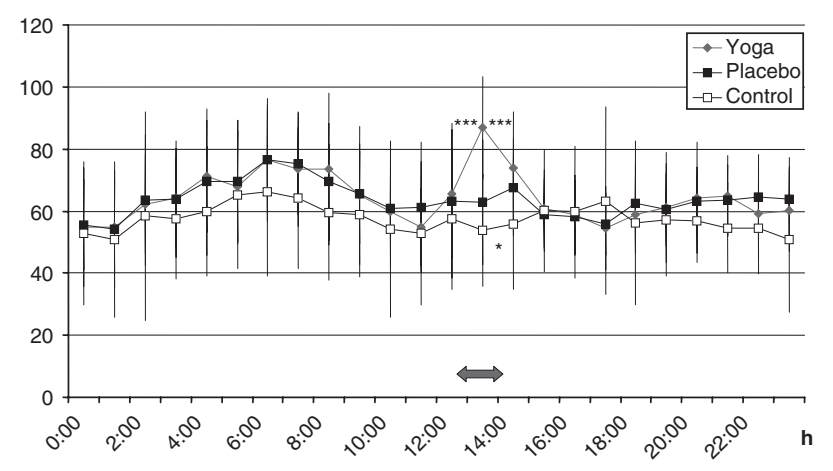

(e) SDANN

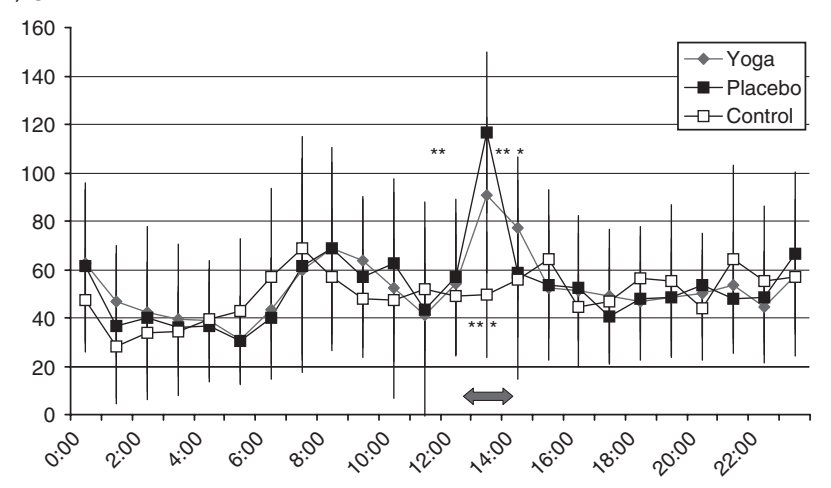

(b)

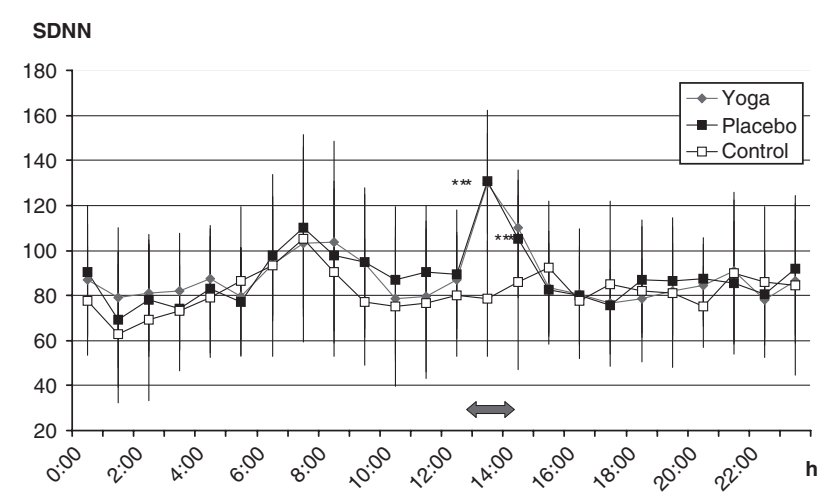

(d)

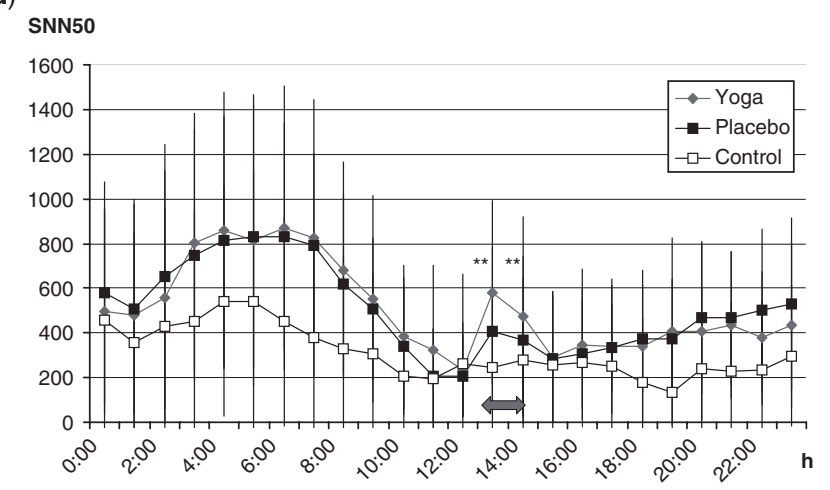

(f) rmssd

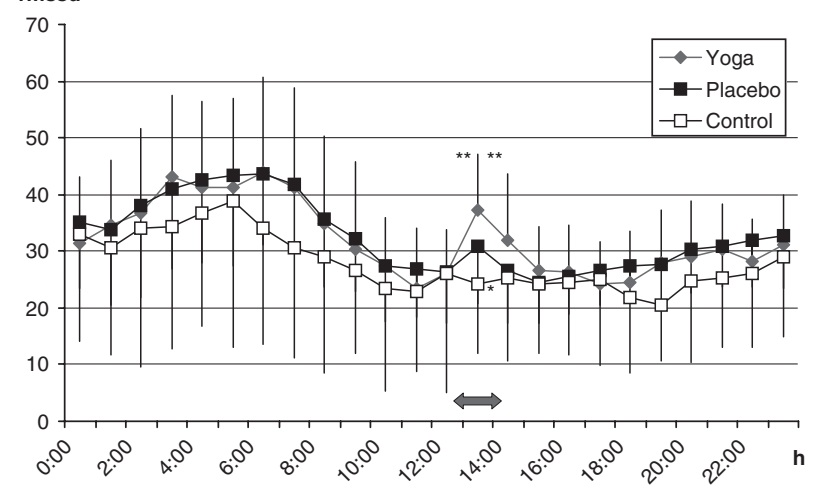

(g) Triangular Index

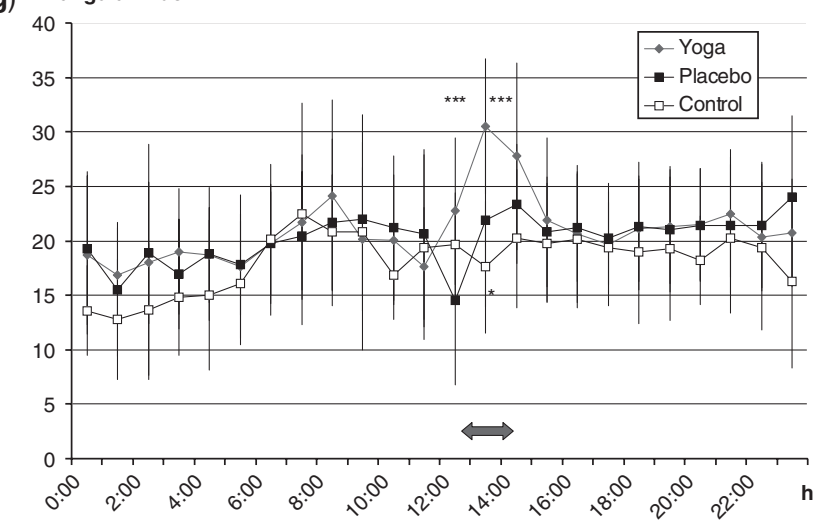


Table 3. Results during time of intervention

\begin{tabular}{|c|c|c|c|c|c|c|}
\hline & $\begin{array}{l}\text { Yoga } \\
\text { mean } \pm \mathrm{SD}\end{array}$ & $\begin{array}{l}\text { Placebo } \\
\text { mean } \pm \text { SD }\end{array}$ & $\begin{array}{l}\text { Control } \\
\text { mean } \pm \mathrm{SD}\end{array}$ & $\begin{array}{l}\text { Significance } \\
\text { yoga versus } \\
\text { placebo }\end{array}$ & $\begin{array}{l}\text { Significance } \\
\text { yoga versus } \\
\text { control }\end{array}$ & $\begin{array}{l}\text { Significance } \\
\text { placebo versus } \\
\text { control } \\
\end{array}$ \\
\hline RR Interval & $864.5 \pm 119$ & $746.4 \pm 86$ & $743.6 \pm 115$ & $P<0.001$ & $P<0.01$ & n.s. \\
\hline SDNNi & $86.9 \pm 16$ & $62.9 \pm 29$ & $53.9 \pm 18$ & $P<0.001$ & $P<0.001$ & $P<0.05$ \\
\hline SNN50 & $580.3 \pm 410$ & $408.7 \pm 371$ & $246.1 \pm 300$ & $P<0.01$ & $P<0.01$ & n.s. \\
\hline TI & $26.5 \pm 6$ & $24.6 \pm 8$ & $17.6 \pm 6$ & $P<0.001$ & $P<0.001$ & $P<0.05$ \\
\hline
\end{tabular}

survival. It was only the combination of exercise with an increase in baroreflex sensitivity that predicted better survival.

From experiments with dogs, Billmann et al. (11) considered the possibility that even independently from physical training, increased baroreflex sensitivity would be associated with a reduced risk for cardiac mortality after myocardial infarction. Also in experiments with dogs after healed myocardial infarction, Kukielka et al. (12) reported that submaximal long-duration exercise reduced cardial vagal regulation initially, but further exercise training attenuated the initially exercise-induced reductions in heart rate variability, suggesting a maintained higher cardiac vagal activity during exercise in the trained state. On the other hand, Duru et al. (13) found no significant effect of high-intensity exercise training on HRV indexes among patients with new-onset left ventricular dysfunction after myocardial infarction after 1,2 and 12 months of training in a rehabilitation center, despite beneficial effects on clinical variables.

These findings suggest, that after myocardial infarction with resulting left ventricular dysfunction which makes up most of post-infarction patients, exercise training remains of limited value on HRV improvements. Furthermore, it seems that in a previously untrained condition, the subject needs to first develop a trained state, before exercise training can have a positive influence on cardial vagal regulation, and that during the phase of building-up exercise capacity an unwanted counter effect might occur. There is a risk that especially young and untrained patients tend to go beyond their (cardiac) exercise capacity. We also experience patients having reduced left ventricular function or comorbidities, who cannot tolerate regular exercise on bicycle ergometry. In these cases, training by relaxation programs could be a suitable alternative and are already established in many rehabilitation centers as an additional training.

A systematic meta-analysis about relaxation therapy for rehabilitation and prevention in ischemic heart disease by Van Dixhoorn and White (14) of 27 controlled trials in which patients with myocardial infarction were taught relaxation therapy revealed that intense supervised relaxation practice enhances recovery from an ischemic cardiac event. This meta-analysis included relaxation techniques such as progressive muscle relaxation, autogenic training, biofeedback, breath relaxation, hypnosis and psychological training. Among these 27 controlled trials, three studies investigated and revealed a positive effect on HRV (15-17). The applied techniques of relaxation were progressive muscle relaxation, breath relaxation, deep breathing, cue controlled relaxation and biofeedback. In our study, we could show that training after the method of B.K.S. Iyengar among healthy yoga practitioners was superior to a simple relaxation program that consisted of resting on the floor and mild exercise like park walking. We think that his method might be superior to other relaxation techniques since it is a unique combination of relaxation [achieved by components like muscle stretching and relaxing, deep breathing, awareness (comparable to biofeedback), psychological aspects (18) concentration and meditation] and very exact therapeutical physical work that can be tailored for any limiting condition or comorbidity. The postures for cardiac patients are chosen and modified in a way to improve the loading-unloading conditions of the heart, which could positively influence remodeling and healing. In every asana, the chest is kept open to improve respiration and achieve a higher oxygenation of blood. Backbending actions give a lengthwise stretch to the mediastinum. Depending on the stage of recovery and condition of the patient, the body is gradually brought to more inverted postures which increase venous return to the heart.

During yoga, the trainees had a lower heart rate than during the alternative program. Even during postures that build up body tension like standing poses or backbends, using slow and more isometric muscle contraction, the heart rate did not rise much. A slow heartbeat prolongs the diastolic filling of the heart, decreases myocardial oxygen consumption and increases myocardial perfusion. A study among 24.913 patients by Diaz et al. (19) clearly identified a high resting heart rate (which also reflects cardial autonomic imbalance towards sympathetic activity) in patients with suspected or proved 
coronary artery disease as an independent predictor for total and cardiovascular mortality. Furthermore, a recently published study introducing deceleration capacity of heart rate - a novel Holter-ECG-based marker for vagal activity - underlines the crucial role of cardiac vagal modulation regarding cardiovascular mortality in post-infarction patients undergoing modern treatment, particularly treatment involving acute revascularization procedures (20).

Further studies are required to investigate whether the demonstrated positive effect of therapeutic yoga on the cardiac vagal modulation can be transferred to cardiac patients and introduced into cardiac rehabilitation programs.

\section{Limitations}

The cohort (regular yoga practitioners) warrants drawing conclusions about long-lasting effects of yoga on HRV parameters. However, the $24 \mathrm{~h}$ circadian rhythm of the yoga practitioners e.g. for $\mathrm{SDNNi}$ and $\mathrm{rMSSD}$ was higher than in the control group, yet the population was too small to show a significant difference.

Additional studies are required to investigate long-term effects of yoga training on cardiac autonomic nervous modulation.

\section{Acknowledgements}

We like to thank Mr B.K.S. Iyengar for his guidance during this study. He tailored the sequence of asanas for this investigation and gave us a glimpse of his knowledge and experience of therapeutic yoga.

\section{References}

1. Ewing DJ, Neilson JMM, Travis P. New method for assessing parasympathetic activity using 24 hour electrocardiograpms. Br Heart J 1984;52:396-402.

2. Task Force of the European Society of Cardiology and the North American Society of Pacing and Electrophysiology. Heart rate variability: standards of measurement, physiological interpretation, and clinical use. Circulation 1996;93:1043-65.

3. Malik M, Camm AJ. Heart rate variability. Clin Cardiol 1990;13:570-6.

4. Kleiger RE, Miller JP, Gigger JT, Moss AJ, and the Multicenter Post-Infarction Research Group. Decreased heart rate variability and its association with increased mortality after acute myocardial infarction. Am J Cardiol 1987;59:256-62.

5. Malik M, Farrell T, Cripps T, Camm AJ. Heart rate variability in relation to prognosis after myocardial infarction. Selection of optimal processing techniques. Eur Heart $J$ 1989;10:1060-74.

6. Bigger JT Jr, Kleiger RE, Fleiss JL, Rolnitzky LM, Steinmann RC, Miller JP, and the Multicenter Post-Infarction Research Group. Components of heart rate variability measured during healing of acute myocardial infarction. Am J Cardiol 1988;61:208-15.

7. Bonnemeier H, Wiegand UKH, Brandes A, Kluge N, Katus HA, Richardt $\mathrm{G}$, et al. Circarian profile of cardiac autonomic nervous modulation in healthy subjects: differing effects of aging and gender on heart rate variability. J Cardiovasc Electrophysiol 2003;14:1-9.

8. Iyengar BKS. Yoga-The path to holistic health. London: Dorling Kindersley Limited, 2001.

9. Iyengar BKS. Light on Yoga. Great Britain: George Allen \& Unwin, 1966.

10. La Rovere MT, Bersano C, Gnemmi M, Specchia G, Schwartz PJ. Exercise-induced increase in baroreflex sensitivity predicts improved prognosis after myocardial infarction. Circulation 2002;106:945-9.

11. Billmann GE, Schwartz PJ, Stone HL. The effects of daily exercise on susceptibility to sudden cardiac death. Circulation 1984;69:1182-9.

12. Kukielka M, Seals DR, Billmann GE. Cardiac vagal modulation of heart rate during prolonged submaximal exercise in animals with healed myocardial infarctions: effects of training. Am J Physiol Heart Circ Physiol 2005;290:H1680-5.

13. Duru F, Candinas R, Dziekan G, Goebbels U, Myers J, Dubach P. Effect of exercise training on heart rate variability in patients with new-onset left ventricular dysfunction after myocardial infarction. Am Heart J 2000;140:157-61.

14. Van Dixhoorn J, White A. Relaxation therapy for rehabilitation and prevention in ischaemic heart disease: a systematic review and metaanalysis. Eur $J$ Cardiovasc Prev Rehabil 2005; 12:193-202.

15. Del Pozo JM, Gervirtz RN, Scher B, Guarneri E. Biofeedback treatment increases heart rate variability in patients with known coronary artery disease. Am Heart J 2004;E11:G1-8.

16. Hase S, Douglas A. Effects of relaxation training on recovery from myocardial infarction. Aust $J$ Adv Nurs 1987;5:18-27.

17. Van Dixhoorn J. Cardiorespiratory effects of breathing and relaxation in myocardial infarction patients. Biol Psychol 1998;49:123-35.

18. Shapiro D, Cook IA, Davydov DM, Ottaviani C, Leuchter AF, Abrams M. Yoga as a complementary treatment of depression: effects of traits and moods on treatment outcome. eCAM, February 28, 2007; doi: doi:10.1093/ecam/nel114.

19. Diaz A, Bourassa MG, Guertin MC, Tardif JC. Longterm prognostic value of resting heart rate in patients with suspected or proven coronary artery disease. Eur Heart $J$ 2005;26:943-5.

20. Bauer A, Kantelhardt JW, Barthel P, Schneider R, Mäkikallio T, Ulm K, et al. Deceleration capacity of heart rate as a predictor of mortality after myocardial infarction: cohort study. Lancet 2006;351:478-84.

Received October 22, 2006; accepted April 23, 2007 


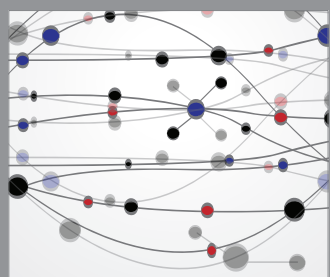

The Scientific World Journal
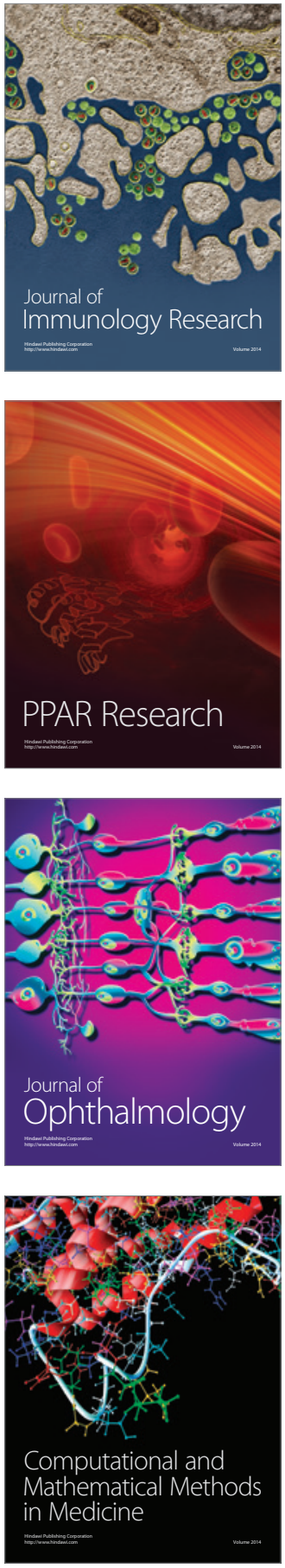

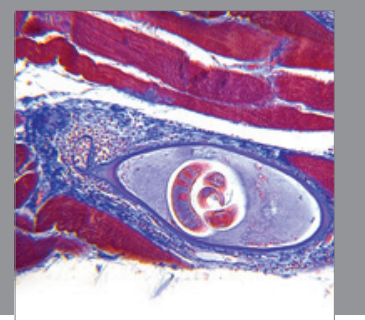

Gastroenterology

Research and Practice
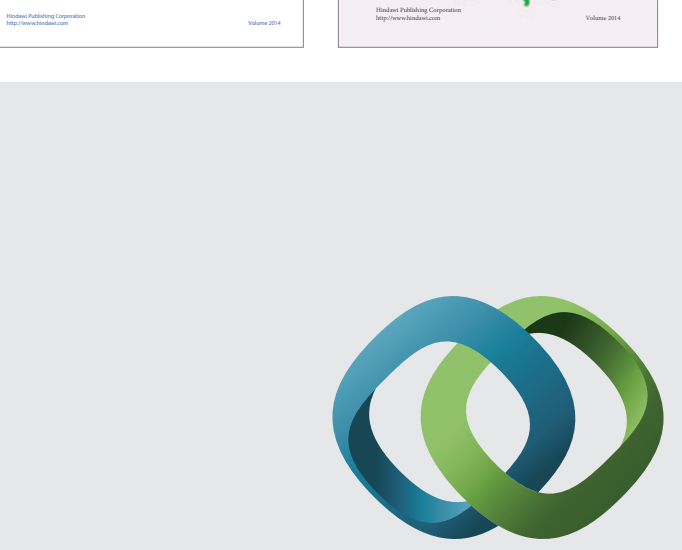

\section{Hindawi}

Submit your manuscripts at

http://www.hindawi.com
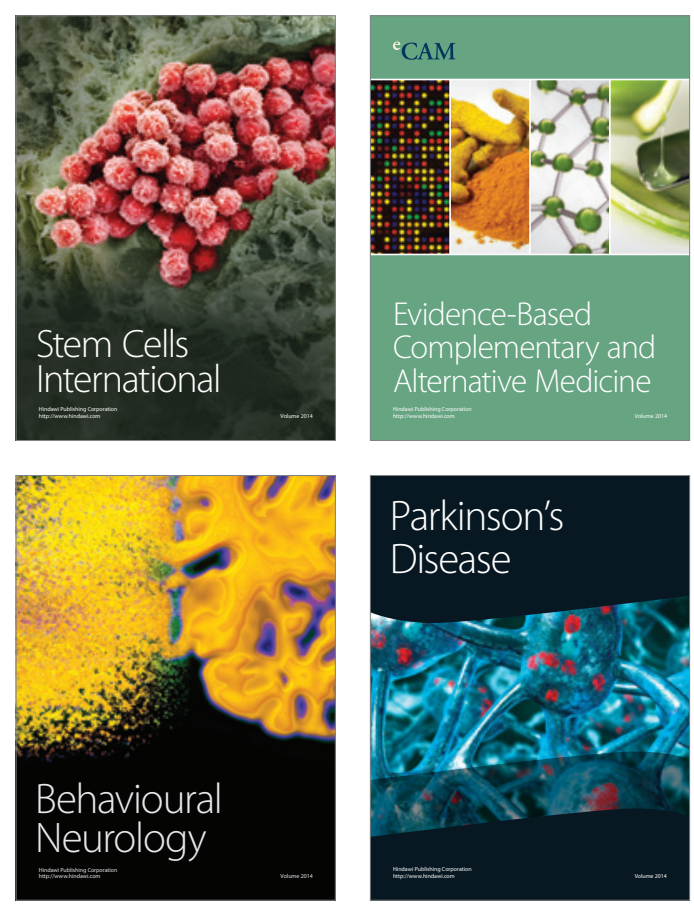

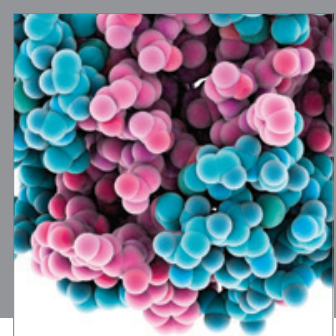

Journal of
Diabetes Research

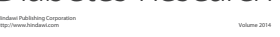

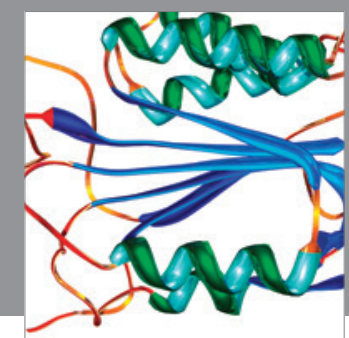

Disease Markers
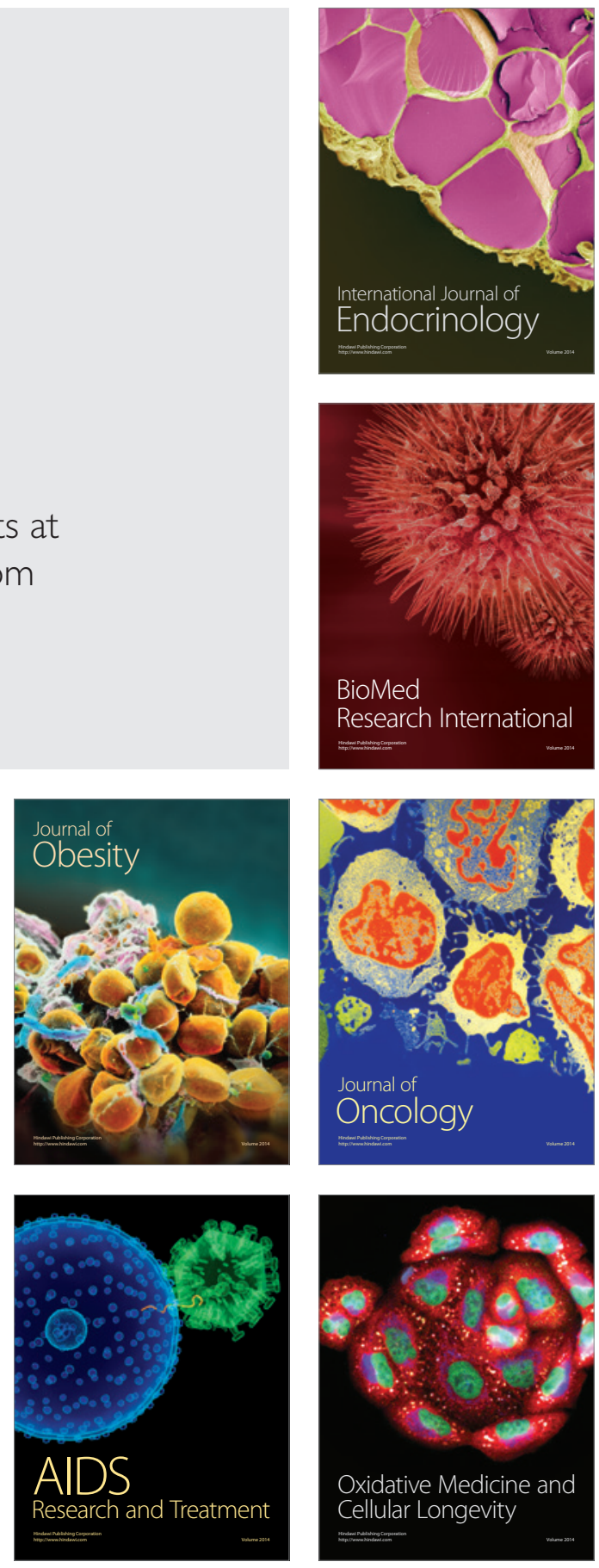